\title{
Das multikulturelle Seniorenzentrum „Haus am Sandberg“
}

\section{Bengi Azcan}

Sozialarbeiterin im Haus am Sandberg, DRK-Landesverband Nordrhein e.V., Duisburg

Das multikulturelle Seniorenzentrum „Haus am Sandberg “ wurde im Januar 1997 bezogen. Es ist die Nachfolgeeinrichtung des DRK-Altenheims „Haus Rheinkamp“.

Bereits im Jahre 1994 wurde ein Pilotprojekt, ESA - Ethnischer Schwerpunkt Altenhilfe, zur Konzipierung eines „internationalen Altenheims“ gestartet. In Kooperation zwischen dem DRKLandesverband Nordrhein e.V., Träger der Einrichtung, und dem Rhein-Ruhr-Institut für Sozialforschung und Politikberatung an der GerhardMercator-Universität Duisburg wurde das dreijährige Projekt, gefördert durch die Stiftung Wohlfahrtspflege, durchgeführt.

Schwerpunkt war unter anderem die interkulturelle Organisationsentwicklung, das heißt die Belegschaft hat sich interkulturalisiert und die Dienstleistungen ebenso. Durch den langjährigen gemeinsamen Lernprozess hat sich ein kollektiv gebildetes Sinnsystem ergeben, was als Handlungsmuster nach außen und Sozialisationskraft nach innen wirkt.

Die vollstationäre Einrichtung verfügt über 96 Plätze in 48 Einzel- und 24 Doppelzimmern. Es gibt auch die Möglichkeit der Kurzzeitpflege.

Das Durchschnittsalter der Bewohner(innen) ohne Zuwanderungsgeschichte liegt bei ca. 83 Jahren, bei Bewohner(inne)n mit Zuwanderungsgeschichte bei ca. 65 Jahren. Die durchschnittliche Verweildauer beträgt fünf Jahre. Zur Zeit leben 17 türkische, ein tunesischer und eine spanische Bewohner(in) im Haus am Sandberg. In den zehn Jahren wurden insgesamt 70 ältere pflegebedürftige Menschen aus anderen Ländern und Kulturen in der Einrichtung betreut. 12 Menschen mit einer Zuwanderungsgeschichte warten zur Zeit auf einen Heimplatz.

In der Einrichtung arbeiten insgesamt ca. 90 Mitarbeiter(innen) (verteilt auf 46 Vollzeitstellen), zuzüglich fünf Zivildienstleistende, drei junge Menschen, die ein Freiwilliges Soziales Jahr leisten, und 60 Ehrenamtliche. Neben 14 türkischstämmigen Mitarbeiter(inne)n beschäftigt die Einrichtung Mitarbeiter(innen) aus Russland, Kasachstan, Polen, den Niederlanden und Italien.
Während die Pflegekräfte mit Zuwanderungsgeschichte zu Beginn noch oft gering qualifiziert waren und in Aushilfspositionen arbeiteten, förderte die Einrichtung in den vergangenen Jahren ihre Ausbildung zu Fachkräften. Zur Zeit sind sieben Auszubildende im Pflegebereich beschäftigt, die aus Russland, der Türkei, Kasachstan und Deutschland kommen. Zwei Wohnbereichsleiter(innen) mit Zuwanderungsgeschichte leiten zur Zeit jeweils einen Wohnbereich, eine Diplom-Sozialwissenschaftlerin mit Zuwanderungsgeschichte ist in der sozialen Betreuung tätig.

Die Leitung bietet ihren Mitarbeiter(inne)n regelmäßig multikulturelle Fortbildungen an, zum Beispiel Sprachkurse und landeskundliche Kurse. Die Teams erhalten bei Bedarf spezielle Supervisionen. Kulturelle Fragestellungen bilden dabei den Schwerpunkt.

Die Einrichtung bietet den Bewohner(inne)n und Angehörigen kulturelle Angebote. Ein interkultureller Besuchsdienst, Gebetsräume für Chris$\mathrm{t}$ (inn)en und Muslim(innen)(e), eine internationale Bibliothek, ein wöchentlicher mediterraner Markt, die Ausrichtung von internationalen Festen gehören dazu.

In den vergangenen Jahren waren mehr als 800 Besuchergruppen aus aller Welt zu Besuch im Multikulturellen Seniorenzentrum in Duisburg. Wir hoffen, allen Gruppen/Personen Anregungen und Mut mit auf den Weg gegeben zu haben, um den Prozess der interkulturellen Öffnung von vollstationären Altenhilfeeinrichtungen in ganz Deutschland voranzutreiben und zu verwirklichen.

Bei allen Besucher(inne)n wurde auch das weitere inhaltliche Konzept, den Wohnaspekt in den Vordergrund zu stellen, einhellig als zukunftsweisender Weg in eine humane Betreuung und Pflege älterer Menschen gelobt. Der kontinuierliche Prozess, alle sichtbaren, an ein Krankenhaus erinnernden Symbole - wie beispielsweise weiße Dienstkleidung oder Begriffe wie Patient/Schwester/Station - durch alltägliche Formen/Symbole wie farbige Bekleidung der Mitarbeiter(innen), Teppiche in den Fluren, Ansprache als Bewohner(in)/Herr/Frau und Mitarbeiter(in)/Herr/Frau oder die Mitnahme von eigenen Möbeln und Haustieren - zu ersetzen, ist gut vorangeschritten. Auch in einem „normalen Wohnumfeld“ kann eine dem SGB XI entsprechende Pflege und Betreuung möglich sein. 